\title{
Analytical Evaluation of the Hadron Number in Extensive Air Showers for Rising Inelasticity
}

Jiben Sidhanta

Department of Physics, Charuchnadra College, Calcutta-700 029, India

Rajkumar Roychoudhury

Electronics Unit, Indian Statistical Institute, Calcutta-700 035, India

'Z. Naturforsch. 39a, $1172-1174$ (1984); received August 8, 1984

A purely analytical method is presented in this paper for evaluating the average number of hadrons (in an air shower) with a given energy as the inelasticity of the cross-section rises. Our result is found to be in excellent agreement with numerical solutions and also with experimental data, particularly in the high energy range.

\section{Introduction}

In view of its importance for multiparticle production processes in the ultra high energy region the analysis of the hadronic components of extensive air showers (EAS) has become an active field of interest. A crucial point of such an analysis consists in determining the average number of hadrons with a given energy. Unfortunately, most of the studies employ numerical techniques involving a cluttering mass of experimental data and a lot of equipment.

The present paper employs a purely analytical method to determine the average number of hadrons with a given initial energy as the inelasticity of the cross-section rises. In a recent paper [1] a solution for nucleons has been found; in the pion case the diffusion equation itself is somewhat harder but our previous approach works, mutatis mutandis, quite satisfactorily. As in the previous paper [1] we shall follow the rigorous saddle-point method which we developed in a recent work [2] for the inversion of the Mellin transform.

The raison d'être of the present paper will be apparent when one considers the following facts:

In the first place the coupled integro-differential equations that occur in the present problem have prompted one numerical solution after another [3]-[5] although they are by no means intractable analytically. Perusing some of them which are found to be protracted over sixteen odd years, we

Reprint requests to Dr. Roychoudhury, Electronics Unit, Inidan Statistical Institute, 203 Barrackpore Trunk Road, Calcutta-700 035, India. have undertaken here to vindicate the feasibility of a purely analytical solution. For the general reader in particular an analytical solution is always more suggestive of novel ideas than any cut and dried numerical sheet. Secondly, the prevalent numerical solutions employ "stronger" assumptions than we need even in our wholly analytical approach. In a standard numerical calculation [3] the nucleon interaction length is approximated by a constant (see the paragraph preceding Eq. (2.4) of [3]). In another [4] $\lambda_{\pi}$ and $\lambda_{N}$ are accorded identical values throughout. We, however, consider a more general situation in the sense that we do not require $\lambda_{\pi}$ and $\lambda_{N}$ to be equal. Of course, the present work too needs some assumption. We assume $a_{\pi}=a_{\mathrm{N}}$ (see Eqs. (4) $-(6)$ below); but then, this is evidently a more tenable hypothesis than $i_{\pi}=i_{\mathrm{N}}$.

\section{Analytical Evaluation of the Hadron Number}

Consider the relevant integro-differential equations (of diffusion):

$$
\begin{aligned}
\frac{\partial N}{\partial p}= & -\frac{N^{\prime}}{\lambda_{\mathrm{N}}}+\int_{E}^{E_{0}} \frac{N\left(E^{\prime}, P\right)}{\lambda_{\mathrm{N}}} W_{\mathrm{NN}}\left(E^{\prime}, E\right) \mathrm{d} E^{\prime}, \\
\frac{\partial \Pi}{\partial p}= & -\frac{1}{\lambda_{\pi}} \Pi+\frac{B}{E_{p}} \Pi+\int_{E}^{E_{0}} \frac{N\left(E^{\prime}, p\right)}{\lambda_{\pi}} W_{\pi \mathrm{N}}\left(E^{\prime}, E\right) \mathrm{d} E^{\prime} \\
& +\int_{E}^{E_{0}} \frac{\Pi\left(E^{\prime}, p\right)}{\lambda_{\pi}} W_{\pi \pi}\left(E^{\prime}, E\right) \mathrm{d} E^{\prime},
\end{aligned}
$$

where $\lambda_{\text {in, } \pi}$ etc. are written $\lambda_{\pi}$ etc. for simplicity, the rest of the symols being as in [5] and [6]. 
Assuming $B / E_{p} \ll 1$ and setting $\Pi=F_{\pi} e^{-p / \lambda_{\pi}}$ and $p^{\prime}=p / i_{-\pi}(E)$, we reduce (2) into:

$$
\begin{aligned}
\frac{\partial F_{\pi}}{\partial p^{\prime}}= & \int_{E}^{E_{0}} \frac{\lambda_{\pi}(E)}{\lambda_{\mathrm{N}}\left(E^{\prime}\right)} \exp \left\{p / \lambda_{\pi}(E)\right\} F_{\mathrm{N}} \\
& \cdot \exp \left\{-p / \lambda_{\mathrm{N}}\left(E^{\prime}\right)\right\} W_{\pi \mathrm{N}}\left(E^{\prime}, E\right) \mathrm{d} E^{\prime} \\
& +\int_{E}^{E_{0}} \frac{\lambda_{\pi}(E)}{\lambda_{\pi}\left(E^{\prime}\right)} e^{p}\left(\frac{1}{\lambda_{\pi}(E)}-\frac{1}{\lambda_{\pi}\left(E^{\prime}\right)}\right) \\
& \cdot F_{\pi} W_{\pi \pi}\left(E^{\prime}, E\right) \mathrm{d} E^{\prime},
\end{aligned}
$$

where we have written

$$
N\left(E^{\prime}, p\right)=F_{\mathrm{N}}\left(E^{\prime}, p\right) \exp \left\{-p / \lambda_{\mathrm{N}}\left(E^{\prime}\right)\right\} .
$$

We next express the rise of inelasticity in the standard form:

$$
\sigma_{\text {in }}=\sigma^{0}\left(1+a \ln \frac{E}{100}\right),
$$

i.e.

$$
\frac{1}{\lambda_{\pi, \mathrm{N}}}=\left(1+a_{\pi, \mathrm{N}} \ln \frac{E}{100}\right) \frac{1}{\lambda_{\pi, \mathrm{N}}^{0}} .
$$

As noted before, we do not require $\lambda_{\pi}=\lambda_{\mathrm{N}}$ but use the somewhat more generally valid assumption $\alpha_{\pi}=\alpha_{N}=a$ (say).

From (4), writing $X=E / E^{\prime}$, we obtain

and

$$
\frac{\lambda_{\pi}(E)}{\lambda_{\pi}\left(E^{\prime}\right)} \cong 1-a \ln X
$$

Also

$$
\frac{\lambda_{\pi}(E)}{\lambda_{\mathrm{N}}\left(E^{\prime}\right)} \cong \frac{\lambda_{\pi}^{0}}{\lambda_{\mathrm{N}}^{0}}(1-a \ln X) .
$$

$$
\frac{1}{\lambda_{\pi}(E)}-\frac{1}{\lambda_{\mathrm{N}}\left(E^{\prime}\right)} \cong \frac{1}{\lambda_{\pi}^{0}}-\frac{1}{\lambda_{\mathrm{N}}^{0}}+\frac{1}{\lambda_{\pi}^{0}} a \ln X,
$$

the last equation following from a neglect of second order of smallness, viz. $a\left(\frac{1}{\lambda_{\pi}^{0}}-\frac{1}{\lambda_{N}^{0}}\right)$.

Accordingly (3) reduces to $\left(w=E / E_{0}\right)$

$$
\begin{aligned}
\frac{\partial F_{\pi}}{\partial p^{\prime}}\left(E, p^{\prime}\right)= & \frac{\lambda_{\pi}^{0}}{\lambda_{\mathrm{N}}^{0}} \int_{w}^{1} f_{\pi \mathrm{N}}(X) \frac{\mathrm{d} x}{X^{2}}(1-a \ln X) F_{\mathrm{N}} \\
& \cdot \exp \left\{a p^{\prime} \ln X\right\} \cdot \exp \left\{p^{\prime}\left(1-\frac{\lambda_{\pi}^{0}}{\lambda_{\mathrm{N}}^{0}}\right)\right\} \\
& +\int_{w}^{1} f_{\pi \pi}(X) \frac{\mathrm{d} x}{X^{2}}(1-a \ln X) F_{\pi} \\
& \cdot \exp \left\{a p^{\prime} \ln X\right\},
\end{aligned}
$$

where we have introduced a naive form of Feynman scaling typified by

$$
f_{\pi \mathrm{N}}(X)=E W_{\pi \mathrm{N}}\left(E, E^{\prime}\right)=\frac{1}{\sigma_{\text {in }}} \int E \frac{\alpha^{3} \sigma}{\alpha p^{3}} \mathrm{~d} p_{t}^{2} .
$$

Subjecting (8) to Mellin transform (defined by $\left.M_{\pi}=\int_{0}^{\infty} F_{\pi} E^{s} \mathrm{~d} E ; \quad M_{\mathrm{N}}=\int_{0}^{\infty} F_{\mathrm{N}} E^{s} \mathrm{~d} E\right)$, we have for large $E_{0}$,

$$
\frac{\partial M_{\pi}}{\partial p^{\prime}}=\frac{\lambda_{\pi}^{0}}{\lambda_{\mathrm{N}}^{0}} g(s) e^{e p^{\prime}} M_{\mathrm{N}}\left(s, p^{\prime}\right)+M_{\pi}\left(s, p^{\prime}\right) g(s),
$$

where

$$
g(s)=\int_{0}^{1} x^{s+a p^{\prime}-1} f(X)(1-a \ln X) \mathrm{d} x
$$

and

$$
\varepsilon=1-\frac{\lambda_{\pi}^{0}}{\lambda_{\mathrm{N}}^{0}}
$$

Equation (9) may be considered a linear differential equation for $M_{\pi}$ whose solution (ignoring the $p^{\prime}$ dependence of $g(s)$ which is inconsequential in view of the smallness of $a$ ) is given by

$$
\begin{aligned}
M_{\pi}= & C e^{p^{\prime} g(s)}+e^{p^{\prime} g(s)} \frac{\lambda_{\pi}^{0}}{\lambda_{\mathrm{N}}^{0}} \\
& \cdot \int g(s) e^{(\varepsilon-g(s)) p^{\prime}} M_{\mathrm{N}}\left(s, p^{\prime}\right) \mathrm{d} p^{\prime},
\end{aligned}
$$

where $C$ is an arbitrary integration constant.

But

$$
M_{\mathrm{N}}\left(s, p^{\prime}\right)=\int_{0}^{\infty} F_{\mathrm{N}} E^{s} \mathrm{~d} E \cong M_{\mathrm{N}}(s, 0) e^{p^{\prime} \bar{g}(s)},
$$

where $\bar{g}(s)$ is related to the process $N N \rightarrow p+X$. Since $M_{\pi}(s, 0)=0, C$ in (10) can be known.

Also, for $\bar{g}(s)+\varepsilon \ll g(s)$, we have

$$
M_{\pi} \cong \frac{\lambda_{\pi}^{0}}{\lambda_{\mathrm{N}}^{0}} M_{\mathrm{N}}(s, 0)\left[e^{p^{\prime} g(s)}-e^{p^{\prime} \bar{g}(s)}\right] .
$$

Again,

$$
M_{\mathrm{N}} \cong M_{\mathrm{N}}(s, 0) e^{p^{\prime} \bar{g}(s)} .
$$

Hence

$$
M_{\pi}+\frac{\lambda_{\pi}^{0}}{\lambda_{\mathrm{N}}^{0}} M_{\mathrm{N}}=\frac{\lambda_{\pi}^{0}}{\lambda_{\mathrm{N}}^{0}} M_{\mathrm{N}}(s, 0) e^{p^{\prime} \bar{g}(s)} .
$$

What is still left is just the inversion of this Mellin-transformed relation. This is found from our previous work of inversion [2] by a comparison. We 
accordingly find

$$
\begin{aligned}
\Pi+\frac{\lambda_{\pi}^{0}}{\lambda_{\mathrm{N}}^{0}} N= & \frac{\lambda_{\pi}^{0}}{\lambda_{\mathrm{N}}^{0}} \frac{C_{0}}{E} \sqrt{\frac{\pi}{p^{\prime}}} \\
& \cdot \exp \left\{p^{\prime}\left[g\left(s_{0}\right)-1\right] \bar{s}_{0} \ln w\right\} .
\end{aligned}
$$

Also, from another previous work of ours [1],

$N=\frac{\bar{C}_{0}}{E} \sqrt{\frac{\pi}{p^{\prime \prime}}} \exp \left\{p\left[\bar{g}\left(s_{0}\right)-1\right]-\bar{s}_{0} \ln W\right\}$,

where $p^{\prime \prime}=\frac{p}{\lambda_{N}(E)}$ and $s_{0}$ is the saddle-point obtained by solving (1). Note that the elegance of the solution (14) is that the only free parameter here is $C_{0}$; for details of the inversion procedure we allude to our previous works [1,2]. From (14) and (15), then, the total hadron spectrum $\Pi+N$ can easily be obtained. If, however, we use $\lambda_{\mathrm{N}}^{0} \approx \lambda_{\pi}^{0}$, (13) alone would yield the total hadron spectrum:

$\Pi+N=\frac{C_{0}}{E} \sqrt{\frac{\pi}{p^{\prime}}} \exp \left\{p^{\prime}\left[g\left(s_{0}\right)-1\right]-\bar{s}_{0} \ln W\right\}$, where $s_{0}$ is given by $\left.\frac{d g(s)}{\alpha s}\right|_{s=s_{0}}=\frac{\ln W}{p^{\prime}}$ and
$\bar{s}_{0}=s_{0}-a p^{\prime}+1$.

Note that in particular, for the process $N N \rightarrow$ $\Pi^{ \pm}+X, f(X)$ can be simulated by the formula [7] $f(X) \sim A_{0}(a-X)^{4}$.

Then

$$
\begin{aligned}
g(s) & =\int_{0}^{1} X e^{s+a p^{\prime}-1} f(X)(1-a \ln X) \mathrm{d} X \\
& \cong A_{5} B\left(s+a p^{\prime}, s\right),
\end{aligned}
$$

where $B$ is the usual beta function. In that event $S_{0}^{*}$ admits of an approximate representation in terms of beta function as follows:

$$
\left.\frac{\mathrm{d}}{\mathrm{d} s} B\left(s+a p^{\prime}, 5\right)\right|_{s=s_{0}}=\frac{\ln W}{A_{0} p} .
$$

The last expression can be solved by successive approximation.

For comparing the result of our calculation with a standard work [8] we use $a=0.03$ and obtain excellent agreement as evinced by Fig. 1, which moreover compares our result with the numerical calculations [5] especially in the higher reaches of the energy range.

The authors thank Dr. D. P. Bhattacharya who made some references on experimental data available.

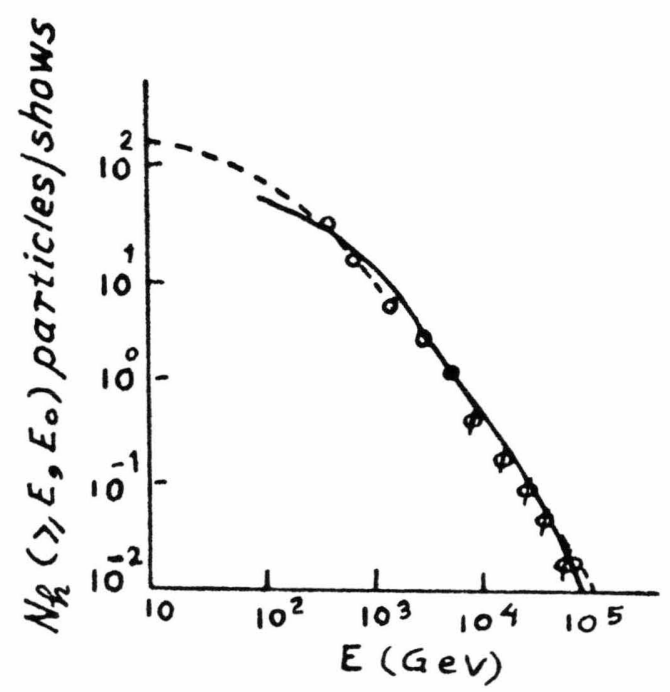

Fig. 1. Energy spectra of hadrons in EAS for $E_{0}=10^{6} \mathrm{GeV}$ and $a=0.03$ at $p=720 \mathrm{gm} / \mathrm{cm}^{2}$. - - - Numerical results of $[5]$; presented work; $\bigcirc$ Dubovoi and Nestero (1975).
[1] J. Sidhanta, R. K. Roychoudhury, Pratibha Pal, and D. P. Bhattacharya, 18th International Cosmic Ray Conf. Bangalore, HE4-40, 5, 368 (1983).

[2] J. Sidhanta, R. K. Roychoudhury, and D. P. Bhattacharya, Phys. Rev. D28, 1113 (1983).

[3] P. M. Fishbane, T. K. Gaisser, R. H. Maurer, and J. S. Trill, Phys. Rev. D9, 3038 (1974).

[4] E. V. Denisov et al., Proc. Int. Conf. Cosmic Rays 1965, HE3. 824 (1965).
[5] M. Boyadzhyan et al., Sov. J. Nucl. Phys. 34 (1), 67 (1981).

[6] A. D. Erlykin, L. K. Ng, and A. W. Wolfendale, J. Phys. A7, 2059 (1974).

[7] J. Nishimura, Handbuch der Physik, S. Flügge (ed.), Springer Verlag, Berlin 1967; Vol. 46, p. 1.

[8] A. Odulovoi and N. N. Nesterov, Brief. Commun. on Phys. No. 8, Phys. Inst. Acad. Sci. USSR 1978, p. 18. 